\title{
Correlation between different instrumentation variants and the degree of destabilization in treating cervical spondylotic spinal canal stenosis by unilateral hemilaminectomy with bilateral decompression: a biomechanical investigation
}

\author{
Ingo Fiss ${ }^{1}\left[\right.$ Dorothee Mielke $^{1} \cdot$ Veit Rohde $^{1} \cdot$ Marios Psychogios $^{2} \cdot$ Christoph Schilling $^{3}$
}

Received: 1 August 2020 / Revised: 19 January 2021 / Accepted: 13 February 2021 / Published online: 10 March 2021

(c) The Author(s) 2021

\begin{abstract}
Purpose Unilateral hemilaminectomy with bilateral decompression (BDZ) was proposed as an alternative decompressive procedure in cervical spondylotic myelopathy (CSM). Despite promising clinical results, the destabilizing effect is yet unknown. We therefore performed a biomechanical study to investigate whether lateral mass screw fixation should follow BDZ.

Methods Six human C2-C7 cervical specimens were tested under various conditions: native, unilateral hemilaminectomy with bilateral decompression without/with fixation (BDZ/BDF), unilateral hemilaminectomy with bilateral decompression and unilateral foraminotomy without/with fixation (UFZ/UFF), unilateral hemilaminectomy with bilateral decompression and bilateral foraminotomy without/with fixation (BFZ/BFF), and laminectomy without/with fixation (LAZ/LAF). Instrumention was applied from C3-C6. For each condition, the three-dimensional kinematics of the cervical specimen were measured in three main loading directions with an ultrasonic motion analysis system. ANOVA was used to determine differences between the specific segment conditions to assess the parameter's range of motion (ROM) and neutral zone (NZ).

Results For flexion-extension, lateral bending and axial rotation, ROM of BDZ, UFZ, BFZ and LAZ remained at the level of the native condition $(p>0.74)$, whereas fixation reduced ROM significantly $(p<0.01)$. Between BDF, UFF, BFF and LAF, no significant differences in reduction in ROM were seen $(p>0.49)$. Results for NZ were equivalent to ROM in flexionextension and lateral bending. For axial rotation, NZ remained almost constant on the native level for all tested conditions. Conclusion Bilateral decompression via a hemilaminectomy, even if combined with foraminotomy, could be a less invasive treatment option for multilevel CSM in patients with lordotic cervical alignment and absence of segmental instability.
\end{abstract}

Keywords Cervical myelopathy $\cdot$ Biomechanics $\cdot$ Lateral mass screw fixation $\cdot$ Less invasive surgery $\cdot$ Unilateral hemilaminectomy

Ingo Fiss

ingo.fiss@med.uni-goettingen.de

Dorothee Mielke

dorothee.mielke@med.uni-goettingen.de

Veit Rohde

veit.rohde@med.uni-goettingen.de

Marios Psychogios

marios.psychogios@usb.ch

Christoph Schilling

christoph.schilling@aesculap.de
1 Department of Neurosurgery, Universitätsmedizin Göttingen, Georg-August University Göttingen, 37099 Göttingen, Germany

2 Department of Neuroradiology, Clinic for Radiology and Nuclear Medicine, University Hospital Basel, 4031 Basel, Switzerland

3 Am Aesculap Platz, Aesculap AG, 78532 Tuttlingen, Germany 


\section{Introduction}

Cervical spondylotic myelopathy (CSM) is a progressive degenerative disease associated with degeneration of intervertebral disks and adjacent vertebral structures, leading to progressive spinal canal narrowing and frequently involving nerve root compression [1-4]. It is the most common cause of myelopathy in patients older than 55 years of age and the leading cause of spinal cord dysfunction in the world [5]. Early surgical treatment can alter the natural history of CSM, as well as improve prognosis in selected patients [6]. Surgical options for decompression of the cervical spinal cord include anterior approaches, such as anterior cervical discectomy and fusion (ACDF) or anterior corpectomy and fusion (ACCF). Laminoplasty (LP) and laminectomy (LAZ) plus fusion are viable posterior alternatives [7]. Despite the higher rate of adjacent segment disease after ACDF and ACCF and identical clinical results, LP is less popular in Europe and in the USA because of an assumed risk of progressive kyphosis due to bilateral muscle detachment [8]. To avoid this, Mielke et al. transferred the concept of achieving bilateral spinal decompression via a unilateral approach, which is today routine in lumbar spinal canals stenosis surgery, to CSM [9-11]. However, it remains unknown, if the benefits of only unilateral muscle detachment are outweighed by a direct destabilizing effect on the cervical spine depending on the amount of bone and yellow ligament removal. The purpose of our in vitro study was to close this knowledge gap and to evaluate the biomechanical changes brought about by this new treatment option using a standardized biomechanical protocol.

\section{Materials and methods}

Six fresh-frozen human cervical spines (C2-C7) with a mean age of 80 years (range $71-90$ years) were kept at $-21{ }^{\circ} \mathrm{C}$ in triple-sealed bags. The spines were thawed overnight at $6{ }^{\circ} \mathrm{C}$ prior to the biomechanical testing. Computed tomography (CT) scans performed in all specimens showed age-related degeneration, however, no fractures, no ossifications of the posterior longitudinal ligament (OPLL), no bridging spondylophytes or syndesmophytes and no osseous ankylosis were revealed. Soft tissue was removed, leaving the ligaments, capsules, thecal sac and supporting structures intact. To fix the specimens firmly in place on the simulator, the cranial and caudal vertebrae ( $\mathrm{C} 2$ and $\mathrm{C} 7$ ) were embedded in a casting resin (Ureol FC 53, Vantico GmbH, Wehr, Germany) in the test fixtures. Segmental motion was not restricted in any way, and the C4-C5 disk was oriented in the horizontal plane. The six specimens were consecutively tested under the following segment conditions: native, after unilateral hemilaminectomy with bilateral decompression without/ with fixation (BDZ/BDF), unilateral hemilaminectomy with bilateral decompression and unilateral foraminotomy without/with fixation (UFZ/UFF), unilateral hemilaminectomy with bilateral decompression and bilateral foraminotomy without/with fixation (BFZ/BFF), and laminectomy without/ with fixation (LAZ/LAF) (Fig. 1).

For lateral mass fixation, 3.5-mm screws (titanium) with a length of 16 or $18 \mathrm{~mm}$ were used (Ennovate Cervical System, Aesculap AG, Tuttlingen, Germany). The instrumentation was performed from C3-C6. To ensure a standardized instrumentation irrespective of the amount of bone removal,
Fig. 1 Cross-sectional sketch depicting the respective segment conditions: a native (NAT); $\mathbf{b}$ unilateral hemilaminectomy with bilateral decompression (BDZ; fixated condition BDF indicated by lateral mass screws); c unilateral hemilaminectomy with bilateral decompression and unilateral foraminotomy (UFZ; fixated condition UFF indicated by lateral mass screws); d unilateral hemilaminectomy with bilateral decompression and bilateral foraminotomy (BFZ; fixated condition BFF indicated by lateral mass screws), e laminectomy (LAZ; fixated condition LAF indicated by lateral mass screws)

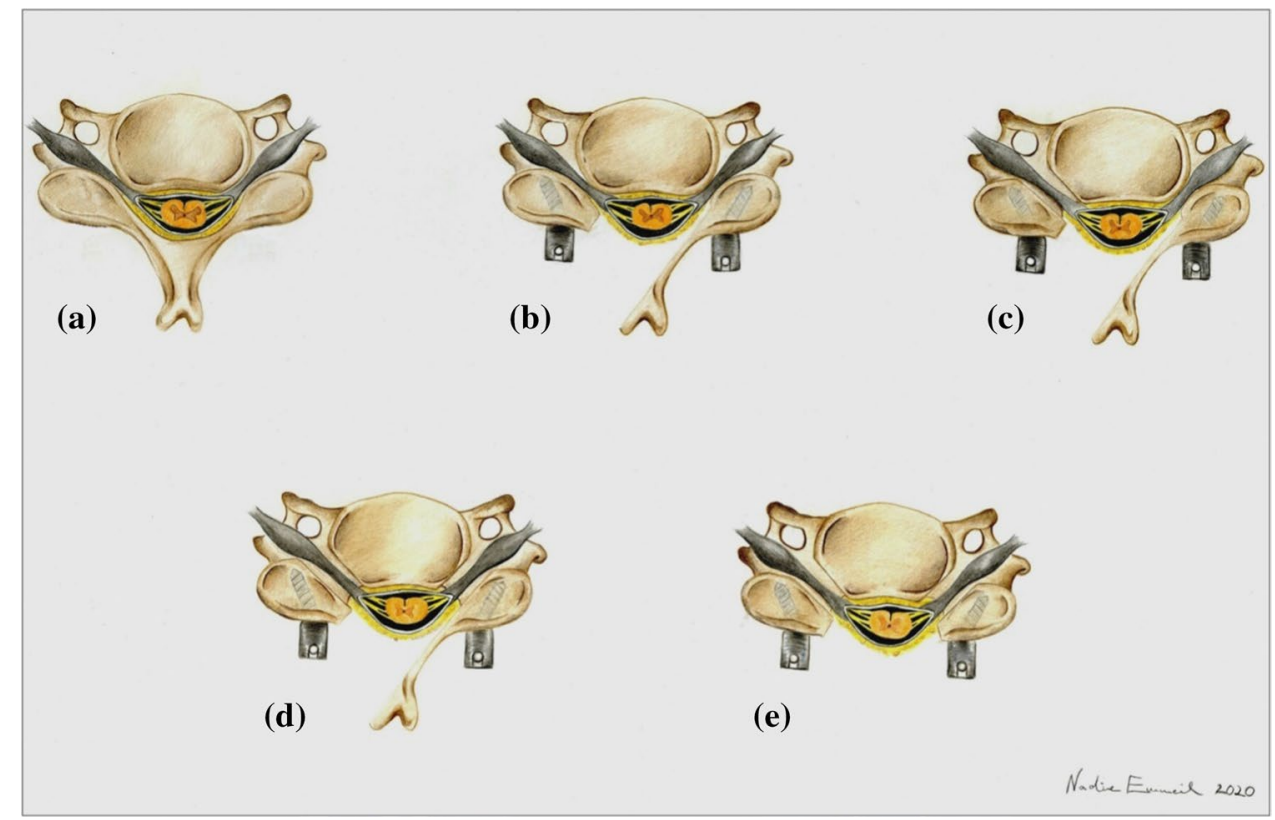


an embedding fixture was used, which kept the initially embedded native segment condition constant. The operations were performed by a board-certified neurosurgeon with ample experience in spine surgery (IF).

The surgical procedure to perform bilateral decompression via hemilaminectomies was as follows (Fig. 1). First, the medial borders of the facet joints were identified, allowing a hemilaminectomy of $\mathrm{C} 4$ and $\mathrm{C} 5$ using a spherical diamond drill and a Kerrison rongeur. Care was taken to preserve the integrity of the facet joints. The next step was the removal of the base of the spinous processes with a 5-mm spherical diamond drill, beginning at the medial edge of the hemilaminectomies and ending near the contralateral medial part of the facet joints, thereby thinning the inner contralateral hemilaminae. Then, bilateral undercutting of the adjacent intact laminae was performed. The yellow ligament was thinned out dorsolaterally in the vicinity of the nerve roots. With a sharp hook, the lateral ligament edge on the side of the approach was elevated. Lastly, the ligament was removed with a Kerrison rongeur until the very first segment of the contralateral dorsal nerve roots was exposed. For the following segment conditions UFZ and BFZ, a foraminotomy was added uni- or bilaterally. Finally, for the segment condition LAZ, laminectomy of C4 and C5 was performed using a Kerrison Rongeur and a diamond drill to remove the contralateral hemilaminae and spinous processes of the involved segments.

For stabilization, the facet joints and lateral masses were identified, and screws were placed bilaterally with the technique described by Magerl et al. [12]. Rods of appropriate size were selected and bent to match the contour of the lateral masses and secured by set screws (segment conditions BDF, UFF, BFF, LAF). Starting with the native condition, measurements were performed after each subsequent step of bony removal, without and with stabilization. The sequence of measurements is depicted in Fig. 2.

The test method complies with the testing criteria for spinal implants [13]. The specimens were loaded with \pm 2.5 $\mathrm{Nm}$ for flexion/extension, lateral bending and axial rotation at room temperature on a spinal simulator based on the principles of Crawford et al. [14]. The load cycle consists of a displacement-controlled loading phase with velocity of $3 \%$ due to the laxity of the specimen followed by a load-controlled phase starting at $2 \%$ below the maximum load $(2.5$ $\mathrm{Nm}$ ) which was kept constant for $1 \mathrm{~s}$ once the maximum load $(2.5 \mathrm{Nm})$ was reached. The kinematics, i.e., the six components of motion according to Panjabi [15], were measured with a non-contacting three-dimensional ultrasonic motion analysis system (Zebris, Isny, Germany) across the entire non-embedded, free length of the specimen. The characteristic parameters range of motion (ROM) and neutral zone (NZ) were analyzed from the hysteresis curves of the third loading cycle, according to Wilke et al. [13].

For statistical analyses on ROM and NZ to determine differences between the specific segment conditions, repeatedmeasures analysis of variance followed by a post hoc test (Least Significance Difference Test) was performed. Prior to analysis, the normal distribution of the data (p-p plots) and the homogeneity of variance (Levene test) were verified. A significance level of $p=0.05$ was defined, and all statistical analyses were performed using Statistica 13 (TIBCO Software, Inc.).

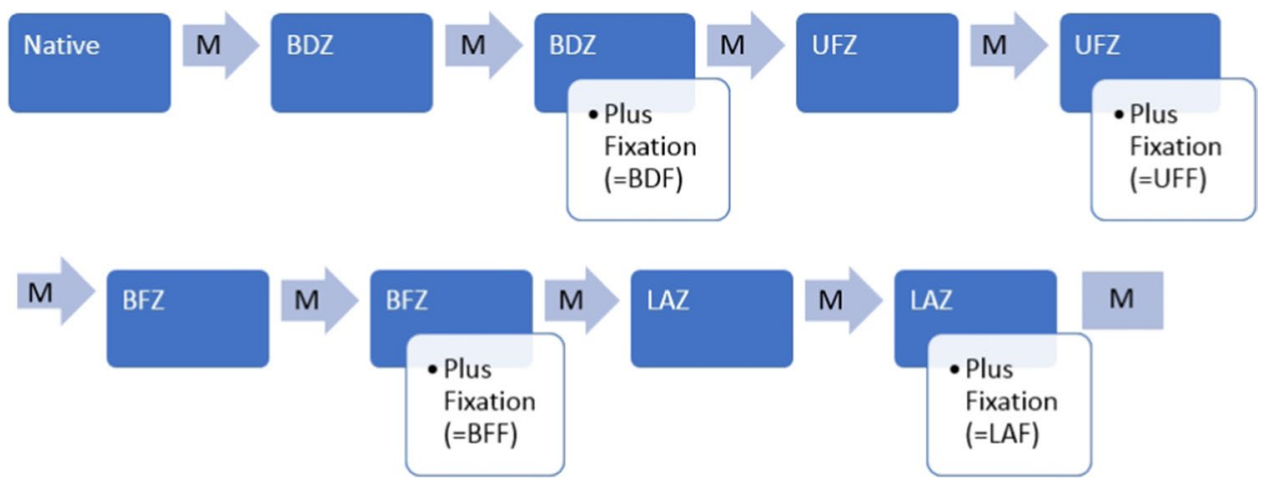

Fig. 2 Flowchart depicting the sequence of measurements of the single segment conditions. ( $M$ measurement, $B D Z$ unilateral hemilaminectomy with bilateral decompression; $B D F$ unilateral hemilaminectomy with bilateral decompression + fixation, $U F Z$ unilateral hemilaminectomy with bilateral decompression and unilateral foraminotomy, UFF unilateral hemilaminectomy with bilateral decompres- sion and unilateral foraminotomy + fixation, $B F Z$ unilateral hemilaminectomy with bilateral decompression and bilateral foraminotomy, $B F F$ unilateral hemilaminectomy with bilateral decompression and bilateral foraminotomy + fixation, $L A Z$ laminectomy, $L A F$ laminectomy + fixation) 


\section{Results}

The ROM and NZ results for all tested segment conditions are summarized in Table 1. For better visualization of the data, the ROM and NZ results (Fig. 3) of the tested conditions are depicted relative to the native condition (NAT), where the NAT is equal to $100 \%$.

For flexion/extension, the total ROM of BDZ $(-9.3 \%$ difference to native condition), UFZ $(-7.1 \%)$, BFZ $(-3.7 \%)$ and LAZ $(-1.4 \%)$ remained at the level of native condition $(p>0.74)$, whereas instrumentation (BDF [-61.3\%], UFF [-57.3\%], BFF [ $-55.3 \%]$ and LAF $[-54.4 \%])$ led to significant reduction in $\operatorname{ROM}(p<0.01)$ without significant differences between BDF, UFF, BFF and LAF, respectively ( $p>0.70)$ (Fig. 3).

For lateral bending, the total ROM of BDZ $(-7.6 \%$ difference to native condition), UFZ (-9.6\%), BFZ (-2.8\%) and LAZ $(-1.3 \%)$ remained at the level of native condition $(p>0.62)$, whereas instrumentation (BDF [-58.8\%], UFF [ $-56.1 \%]$, BFF [ $-55.0 \%]$ and LAF [ $-53.0 \%])$ led to significant reduction in $\operatorname{ROM}(p<0.01)$ without significant differences between BDF, UFF, BFF and LAF, respectively $(p>0.71)$ (Fig. 3).

For axial rotation, the total ROM of BDZ $(-6.2 \%$ difference to native condition), UFZ $(-10.7 \%)$, BFZ $(+3.4 \%)$ and LAZ $(+7.8 \%)$ remained at the level of native condition $(p>0.59)$, whereas instrumentation (BDF [ $-66.0 \%]$, UFF

Table 1 Summary of range of motion (ROM) and neutral zone (NZ) results of all tested segment conditions

\begin{tabular}{|c|c|c|}
\hline Condition & $\begin{array}{l}\mathrm{ROM}( \pm \mathrm{SD})\left[^{\circ}\right] \\
\text { Flexion/extension }(\mathrm{FE})\end{array}$ & $\begin{array}{l}\mathrm{NZ}( \pm \mathrm{SD})\left[{ }^{\circ}\right] \\
\text { Flexion/extension }(\mathrm{FE})\end{array}$ \\
\hline NAT & $15.6( \pm 6.2)$ & $7.7( \pm 5.0)$ \\
\hline $\mathrm{BDZ}$ & $14.6( \pm 6.8)$ & $8.8( \pm 5.0)$ \\
\hline $\mathrm{BDF}$ & $6.2( \pm 3.0)$ & $3.2( \pm 2.2)$ \\
\hline UFZ & $14.7( \pm 6.2)$ & $7.8( \pm 4.6)$ \\
\hline UFF & $6.8( \pm 3.0)$ & $3.5( \pm 2.5)$ \\
\hline BFZ & $15.2( \pm 6.4)$ & $8.9( \pm 4.8)$ \\
\hline $\mathrm{BFF}$ & $7.2( \pm 3.3)$ & $3.9( \pm 3.1)$ \\
\hline LAZ & $15.7( \pm 6.7)$ & $7.0( \pm 4.5)$ \\
\hline LAF & $7.4( \pm 3.5)$ & $3.9( \pm 2.8)$ \\
\hline \multirow[t]{2}{*}{ Condition } & $\mathrm{ROM}( \pm \mathrm{SD})\left[^{\circ}\right]$ & $\mathrm{NZ}( \pm \mathrm{SD})\left[{ }^{\circ}\right]$ \\
\hline & Lateral bending (LB) & Lateral bending (LB) \\
\hline NAT & $11.1( \pm 3.4)$ & $5.6( \pm 2.7)$ \\
\hline $\mathrm{BDZ}$ & $10.6( \pm 4.7)$ & $5.9( \pm 3.6)$ \\
\hline $\mathrm{BDF}$ & $4.6( \pm 1.4)$ & $2.2( \pm 1.5)$ \\
\hline UFZ & $10.2( \pm 3.8)$ & $5.7( \pm 3.1)$ \\
\hline UFF & $4.8( \pm 1.1)$ & $2.2( \pm 1.6)$ \\
\hline $\mathrm{BFZ}$ & $10.9( \pm 3.8)$ & $6.0( \pm 3.3)$ \\
\hline $\mathrm{BFF}$ & $4.9( \pm 1.3)$ & $2.3( \pm 1.5)$ \\
\hline LAZ & $11.1( \pm 3.9)$ & $6.1( \pm 3.3)$ \\
\hline LAF & $5.2( \pm 1.5)$ & $2.3( \pm 1.8)$ \\
\hline \multirow[t]{2}{*}{ Condition } & $\mathrm{ROM}( \pm \mathrm{SD})\left[^{\circ}\right]$ & $\mathrm{NZ}( \pm \mathrm{SD})\left[^{\circ}\right]$ \\
\hline & Axial rotation (AR) & Axial rotation (AR) \\
\hline NAT & $11.1( \pm 3.4)$ & $5.6( \pm 2.7)$ \\
\hline $\mathrm{BDZ}$ & $10.6( \pm 4.7)$ & $5.9( \pm 3.6)$ \\
\hline $\mathrm{BDF}$ & $4.6( \pm 1.4)$ & $2.2( \pm 1.5)$ \\
\hline UFZ & $10.2( \pm 3.8)$ & $5.7( \pm 3.1)$ \\
\hline UFF & $4.8( \pm 1.1)$ & $2.2( \pm 1.6)$ \\
\hline $\mathrm{BFZ}$ & $10.9( \pm 3.8)$ & $6.0( \pm 3.3)$ \\
\hline $\mathrm{BFF}$ & $4.9( \pm 1.3)$ & $2.3( \pm 1.5)$ \\
\hline LAZ & $11.1( \pm 3.9)$ & $6.1( \pm 3.3)$ \\
\hline LAF & $5.2( \pm 1.5)$ & $2.3( \pm 1.8)$ \\
\hline
\end{tabular}

Absolute values: mean [ \pm standard deviation $(\mathrm{SD})]$ 

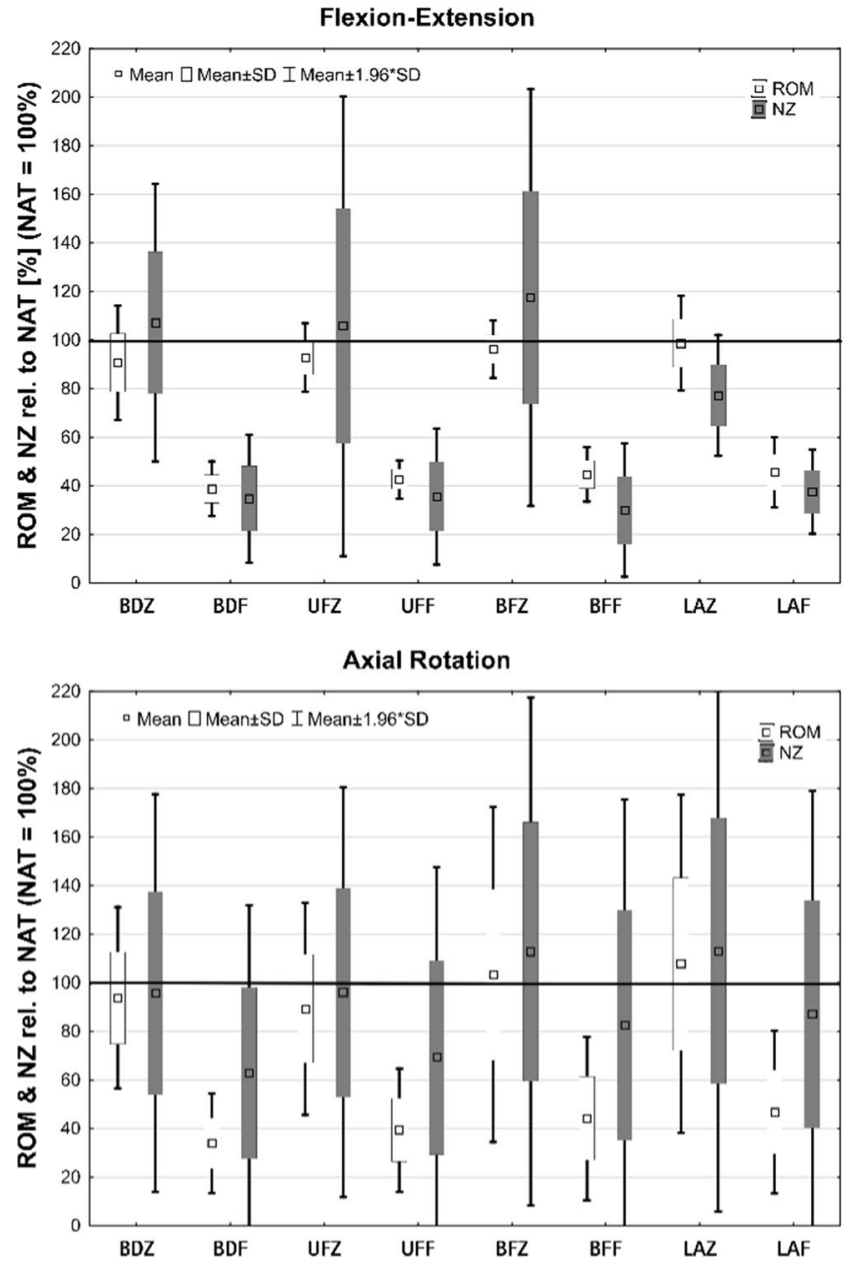

Fig. 3 Range of motion (ROM) and neutral zone (NZ) of the subaxial cervical spine $(\mathrm{C} 2-\mathrm{C} 7)$. The different segment conditions are normalized to the native situation representing $100 \%$. NAT native, $B D Z$ unilateral hemilaminectomy with bilateral decompression, $B D F$ unilateral hemilaminectomy with bilateral decompression + fixation, $U F Z$ unilateral hemilaminectomy with bilateral decompression and unilat-

[-60.7\%], BFF [-55.8\%] and LAF [-53.2\%]) led to significant reductions of $\mathrm{ROM}(p<0.01)$ without significant differences between BDF, UFF, BFF and LAF, respectively $(p>0.49)$ (Fig. 3).

The results for the NZ showed equivalent values compared to ROM in flexion/extension and lateral bending. For axial rotation, the NZ remained almost constant on the native level for all tested segment conditions (Fig. 3).

\section{Discussion}

\section{Key findings}

After encouraging initial clinical results, we replaced the standard anterior and posterior operations for spinal cord

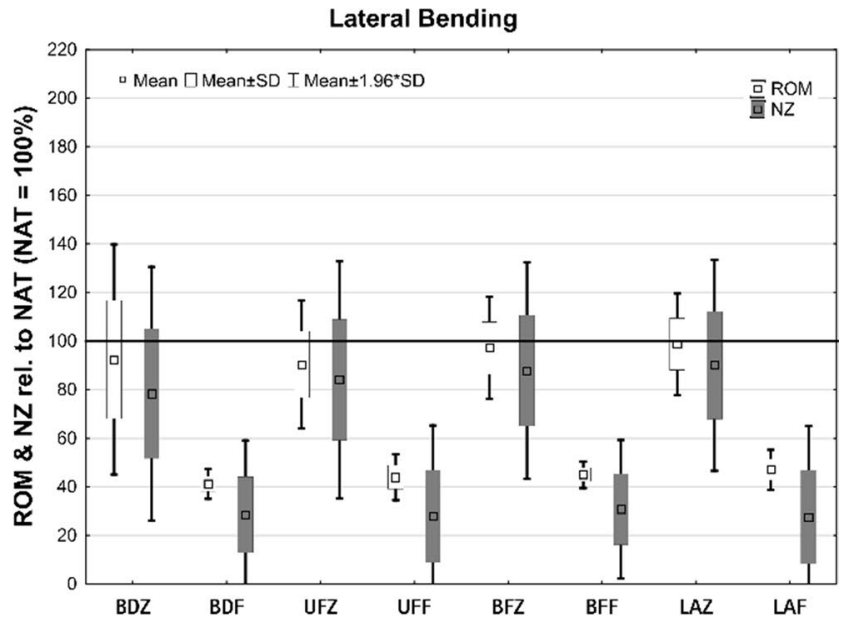

eral foraminotomy, $U F F$ unilateral hemilaminectomy with bilateral decompression and unilateral foraminotomy + fixation, $B F Z$ unilateral hemilaminectomy with bilateral decompression and bilateral foraminotomy, $B F F$ unilateral hemilaminectomy with bilateral decompression and bilateral foraminotomy + fixation, $L A Z$ laminectomy, $L A F$ laminectomy + fixation

decompression in patients with CSM by hemilaminectomy with bilateral decompression (BDZ). As being a new method, long-term follow-up data will not be soon available, and the rate of postoperative loss of lordosis or kyphosis will remain unknown. Until now, an interim analysis of our current prospective study concerned with the clinical evaluation of patients undergoing BDZ (study number 20/2/14) showed improvement in the Neck Disability Index (NDI) without the development of kyphosis in lateral X-ray studies. For knowing if BDZ, especially if combined with uni- or bilateral foraminotomy, might nevertheless have a destabilizing effect on the cervical spine, we decided to conduct a biomechanical study. The key finding is that BDZ, UFZ, BFZ do not decrease the stability of the cervical spine. An interesting finding was that also laminectomy (LAZ) did not produce an increase in the ROM in flexion/extension, lateral 
bending and axial rotation. An expected finding was that instrumentation reduced the ROM significantly.

\section{Experimental protocol}

The experimental protocol applied in this study is based on the current recommendations for in vitro testing of spinal implants [13-15]. The motion measurements were performed by means of a three-dimensional ultrasonic motion analysis system across the non-embedded free length of the specimen (i.e., from $\mathrm{C} 2-\mathrm{C} 7$ ). The embedding fixture enabled us to perform a standardized procedure for the distinct surgical steps from C3-C6. Because we applied pure unconstrained moments, the measurement across the intact disk at the level of $\mathrm{C} 2 / \mathrm{C} 3$ and $\mathrm{C} 6 / \mathrm{C} 7$ is an appropriate method for evaluating the motion behavior of different types of instrumentation between C3 and C6 [16].

\section{Other studies}

Other data derived from in vitro studies on the biomechanical properties of cervical hemilaminectomy are actually scarce. Wu et al. [17] established an animal model in sheep to assess biomechanical changes after unilateral hemilaminectomy and different degrees of facetectomy. They performed no operation (group A), C4-C6 unilateral hemilaminectomy (group B), C4-C6 unilateral hemilaminectomy and partial (50\%) ipsilateral C4-C5 facetectomy (group C), C4-C6 unilateral hemilaminectomy and complete ipsilateral C4-C5 facetectomy (group D) in five sheep, respectively. After 24 weeks, fresh cervical spine specimens were acquired and biomechanically tested. ROM in flexion-extension and lateral bending in group D (unilateral hemilaminectomy and complete ipsilateral $\mathrm{C} 4-\mathrm{C} 5$ facetectomy) was significantly greater than that in group $\mathrm{A}$ and $\mathrm{B}$, whereas ROM in axial rotation in group $D$ was not higher than that in group A and B. They concluded that unilateral hemilaminectomy does not affect cervical stability and that unilateral hemilaminectomy and partial ipsilateral facetectomy do not affect long-term cervical stability, thereby supporting our results. Wu et al. proposed that unilateral hemilaminectomy and complete ipsilateral facetectomy can lead to long-term instability. However, complete facetectomy is not necessary in hemilaminectomy with bilateral decompression for CSM. Xie et al. modified a validated nonlinear finite element model (FEM) of the intact cervical spine (C2-C7) to study the biomechanical changes of multilevel laminectomy, multilevel hemilaminectomy and unilateral multilevel interlaminar fenestration with or without unilateral facetectomy for the treatment of intradural tumors at the levels C3-C6 [18]. The less invasive approaches of unilateral interlaminar fenestration and hemilaminectomy mostly preserved the flexion motion (more than $48 \%$ ) of the cervical spine compared to laminectomy, thus minimizing the risk of postoperative spinal instability and disk degeneration. Hashiguchi et al. performed a biomechanical study of cervical posterior decompression and examined the dynamic characteristics of different operative procedures using three-dimensional FEMs [19]. They created models for the preoperative status, laminectomy, Z-plasty, open door laminoplasty, French open door laminoplasty, en bloc laminoplasty and double-door laminoplasty, respectively. Unfortunately, the less invasive alternative of hemilaminectomy with bilateral decompression was not investigated in their study.

\section{Limitations}

There are some limitations to our study. The method of applying pure moments does not fully reflect physiological loading, as compressive and shear forces cannot be considered. Furthermore, the influence of muscle stability could not be addressed, although it is well known that muscles have a strong impact on the stability of the cervical spine. In addition, we only investigated the primary stability of the different decompression techniques without and with fixation. There might be differences between the various noninstrumented decompression techniques after cyclic loading, which are not obvious after testing for primary stability. This might explain the preservation of spine stability even after laminectomy, because the effects of the removed posterior ligaments will probably only become apparent after extensive cyclic loading. It must also be considered that a change in the number of hemilaminectomies could lead to different biomechanical results. Also, the mean age of the specimen of 80 years may be a minor limitation to the study, as the flexibilty of the spine decreases with increasing age and cervical myelopathy predominantly affects patients in their 7 th decade of life.

Based on our results, we further believe that BDZ is a viable, less invasive treatment option for decompression of CSM in patients with straight or lordotic cervical alignment and absence of segmental instability. If required because of accompanying radiculopathy, uni- or bilateral foraminotomy can be added without risking increased instability of the spinal segment.

\section{Conclusions}

Compared to laminoplasty and laminectomy, BDZ without or with uni- or bilateral foraminotomy might offer advantages, being the avoidance of destabilizing bilateral muscle detachment and the need for implants.

Acknowledgements Implants and instrumentations were provided by Aesculap AG, Tuttlingen, Germany. Correlation between different 
instrumentation variants and the degree of destabilization in treating cervical spondylotic spinal canal stenosis by unilateral hemilaminectomy with bilateral decompression: a biomechanical investigation

Funding Open Access funding enabled and organized by Projekt DEAL.. Not applicable.

Data availability All data generated or analyzed during this study are included in this published article.

\section{Compliance with ethical standards}

Conflict of interest The authors declared that they have no conflict of interset.

Ethical approval The trial was approved by the local ethics committee of the Medical University of Göttingen, Germany (Study Number 22/7/17).

Open Access This article is licensed under a Creative Commons Attribution 4.0 International License, which permits use, sharing, adaptation, distribution and reproduction in any medium or format, as long as you give appropriate credit to the original author(s) and the source, provide a link to the Creative Commons licence, and indicate if changes were made. The images or other third party material in this article are included in the article's Creative Commons licence, unless indicated otherwise in a credit line to the material. If material is not included in the article's Creative Commons licence and your intended use is not permitted by statutory regulation or exceeds the permitted use, you will need to obtain permission directly from the copyright holder. To view a copy of this licence, visit http://creativecommons.org/licenses/by/4.0/.

\section{References}

1. Broughton E (2015) Cervical spondylotic myelopathy. In: Bhatia $\mathrm{R}$, Sabin I (eds) Challenging concepts in neurosurgery: cases with expert commentary. Oxford University Press, Oxford, pp 161-170

2. Liu Y, Hou Y, Yang L, Chen H, Wang X, Wu X, Gao R, Wang C, Yuan W (2012) Comparison of 3 reconstructive techniques in the surgical management of multilevel cervical spondylotic myelopathy. Spine 37:E1450-E1458

3. Lawrence BD, Jacobs WB, Norvell DC, Hermsmeyer JT, Chapman JR, Brodke DS (2013) Anterior versus posterior approach for treatment of cervical spondylotic myelopathy: a systematic review. Spine 38(22 Suppl 1):S173-S182

4. Nurick S (1972) The pathogenesis of the spinal cord disorder associated with cervical spondylosis. Brain 95(1):87-100

5. Karadimas SK, Erwin WM, Ely CG, Dettori JR, Fehlings MG (2013) Pathophysiology and natural history of cervical spondylotic myelopathy. Spine 38:S21-S36

6. Rao RD, Gourab K, David KS (2006) Operative treatment of cervical spondylotic myelopathy. J Bone Jt Surg Am 88:1619-1640
7. König SA, Spetzger U (2014) Modified open-door laminoplasty for the surgical treatment of cervical spondylotic myelopathy in elderly patients. Acta Neurochir 156:1225-1230

8. Ghogawala Z, Martin B, Benzel EC, Dziura J, Magge SN, Abbed KM, Bisson EF, Shahid J, Coumans JV, Choudhri TF, Steinmetz MP, Krishnaney AA, King JT Jr, Butler WE, Barker FG 2nd, Heary RF (2011) Comparative effectiveness of ventral vs dorsal surgery for cervical spondylotic myelopathy. Neurosurgery 68:622-631

9. Mielke D, Rohde V (2015) Bilateral spinal canal decompression via hemilaminectomy in cervical spondylotic myelopathy. Acta Neurochir 157:1813-1817

10. Poletti CE (1995) Central lumbar stenosis caused by ligamentum flavum: unilateral laminotomy for bilateral ligamentectomy: prelimary report of two case. Neurosurgery 37(2):343-347

11. Spetzger U, Bertalanffy H, Reinges MHT, Gilsbach JM (1997) Unilateral laminotomy for bilateral decompression of lumbar spinal stenosis part II: clinical experiences. Acta Neurochir 139(5):397-403

12. Magerl F, Grob D, Seemann PS (1987) Stable dorsal fusion of the cervical spine (C2-T1) using hook plates. In: Kehr P, Weidner A (eds) Cervical spine I. Springer, New York, pp 217-221

13. Wilke HJ, Wenger K, Claes L (1998) Testing criteria for spinal implants: recommendations for the standardization of in vitro stability testing of spinal implants. Eur Spine J 7(2):148-154

14. Crawford NR, Brantley AG, Dickman CA, Koeneman EJ (1995) An apparatus for applying pure nonconstraining moments to spine segments in vitro. Spine 20:2097-2100

15. Panjabi MM, Oxland TR, Yamamoto I, Crisco JJ (1994) Mechanical behavior of the human lumbar and lumbosacral spine as shown by three-dimensional load-displacement curves. J Bone Jt Surg Am 76:413-424

16. Panjabi MM, Malcolmson G, Teng E, Tominaga Y, Henderson G, Serhan H (2007) Hybrid multidirectional test method to evaluate spinal adjacent-level effects. Clin Biomech 22:257-265

17. Wu C, Wang ZY, Lin GZ, Yu T, Liu B, Si Y, Zhang YB, Li YC (2019) Biomechanical changes of sheep cervical spine after unilateral hemilaminectomy and different degrees of facetectomy. Beijing Da Xue Xue Bao Yi Xue Ban 51(4):728-732

18. Xie T, Qian J, Lu Y, Chen B, Jiang Y, Luo C (2013) Biomechanical comparison of laminectomy, hemilaminectomy and a new minimally invasive approach in the surgical treatment of multilevel cervical intradural tumour: a finite element analysis. Eur Spine J 22:2719-2730

19. Hashiguchi A, Kanchiku T, Nishida N, Taguchi T (2018) Biomechanical study of cervical posterior decompression. Asian Spine J 12(3):391-397

Publisher's Note Springer Nature remains neutral with regard to jurisdictional claims in published maps and institutional affiliations. 